\title{
Editorial \\ Computational techniques for applied econometric analysis of macroeconomic and financial processes
}

During recent decades a revolutionary change has taken place in the field of econometric analysis of macroeconomic and financial processes caused by major developments in computational methods. Evaluating many different forecasts and policy scenarios in macroeconomics and computing risk in financial processes is becoming standard practice for government agencies and research divisions of investment banks. Modelling the probability of finding a job and/or getting a loan in terms of explanatory variables such as education, income and age is also common. All these topics require nontrivial computational methods for practical analysis.

The articles in this special issue feature several advances in computational techniques. Four papers refer to developments in simulation methods as described, for example, in the recent textbooks by Koop (2003) and Geweke (2005). There is also strong progress in the frequentist world, where nowadays simulated maximum likelihood and simulated methods of moments estimators are frequently used, see, for example, Gouriéroux and Monfort (1996) and Train (2003). The new developments in simulation methods not only solve many computational problems; they also create new challenging areas for research. Some of these are taken up in the papers collected here.

The common theme of the first three papers is mixture processes. Mixture processes are flexible and tractable structures; they are often used to explain the observed variation in a discrete response variable like buying a house or obtaining a loan in terms of a set of explanatory variables. The papers by Frühwirth-Schnatter and Frühwirth (2007) and by Geweke (2007) show that simple Markov chain Monte Carlo sampling works in finite mixture models. In the first paper a simulation algorithm is proposed for Bayesian analysis of binary and multinomial logit models that requires no tuning parameter. Through the introduction of a data augmentation scheme using two sequences of auxiliary latent variables the authors implement a simple Gibbs sampling scheme. In the second finite mixture paper Geweke emphasizes the distinction between the interpretation of the posterior distribution and the use of simulation methods to represent the posterior. The interpretation becomes difficult when the mixture components have substantive interpretation, whereas it is straightforward when they do not. Geweke shows that conventional Markov chain Monte Carlo methods provide an adequate representation of the posterior distribution and that no special treatment is required in spite of the fact that the posterior is multimodal. Bauwens et al. (2007) make use of mixture processes in the specification of a new multivariate volatility model. The conditional distribution of a vector of financial economic time series is specified by a mixture of multivariate normal distributions where each component may have a time-varying covariance matrix. A simple Griddy Gibbs sampling method is used as posterior simulator and the model is tested on daily US stock returns.

Gelfand et al. (2007) build a spatial model that is analogous to a multilevel model. Their motivation is empirical: model individual preferences with respect to decisions about where to live. In this context one can imagine individuals living or, otherwise stated, nested in a neighbourhood and such neighbourhoods nested in cities. In this way spatial dependence is introduced at several levels in a multilevel model. Data on 68,000 condominium sales across 1374 buildings in Singapore are used for the empirical work. The Markov chain Monte Carlo method constructed by the authors makes use of a multivariate kernel convolution or moving average in order to evaluate the likelihood function. The results obtained illustrate the feasibility of the authors approach. 
The next set of three papers use different computational techniques. Clements and Kim (2007) use bootstrap methods for the evaluation of finite sample performance of frequentist econometric methods. They focus on forecast interval evaluation of three estimators for autoregressive time series that are potentially near-unit root processes. The bootstrap method that uses the Roy-Fuller estimator is preferred overall.

Despite substantial progress in the field of nonlinear optimization, there is a need for advanced optimization methods that can handle investment decisions where the data are erroneous and forecasts are imprecise. Gülpinar and Rustem (2007) describe the mean value of a portfolio in terms of rival returns scenario trees. The authors present a min-max approach for the single-period and multi-period mean-variance optimization problem with multiple rival risks and return scenarios. Their experiments indicate that using rival risk and return forecasts within a robust framework is desirable.

The final paper in this issue is related to simulation in the classic sense. The paper by Heij et al. (2007) compares two methods for dealing with forecasting with many predictors: principal component regression and principal covariate regression. Data are simulated from factor models and regression models. Their simulation results indicate that principal component regressions work better in factor models and principal covariate regressions work better in the other type of data. That is, simulations assist well in the choice between such regression methods in forecasting.

Recently two special issues of Computational Statistics and Data Analysis labelled as: Computational Econometrics (2005) and Nonlinear Modelling and Financial Econometrics (2006) also deal with computational procedures for macroeconomics and finance. Some references to papers that cover similar problems as the papers in this issue are: Bulla and Bulla (2006), Gamerman et al. (2003), Gilli and Winker (2003), Jung et al. (2006), Kim and Wang (2006), Nankervis (2005) and Sanford and Martin (2005).

Finally, the guest editors want to thank all contributors and referees for keeping to a tight time schedule while maintaining high quality manuscripts and referee reports.

\section{References}

Bauwens, L., Hafner, C.M., Rombouts, J.V.K., 2007. Multivariate mixed normal conditional heteroskedasticity. Comput. Statist. Data Anal., this issue, doi:10.1016/j.csda.2006.10.012.

Bulla, I., Bulla, J., 2006. Stylized facts of financial time series and hidden semi-Markov models. Comput. Statist. Data Anal. 51 (4), $2192-2209$.

Clements, M.P., Kim, J.H., 2007. Bootstrap prediction intervals for autoregressive time series. Comput. Statist. Data Anal., this issue, doi:10.1016/j.csda.2006.09.012.

Frühwirth-Schnatter, S., Frühwirth, R., 2007. Auxiliary mixture sampling with applications to logistic models. Comput. Statist. Data Anal., this issue, doi:10.1016/j.csda.2006.10.006.

Gamerman, D., Moreira, A.R.B., Rue, H., 2003. Space-varying regression models: specifications and simulation. Comput. Statist. Data Anal. 42 (3), $513-533$.

Gelfand, A.E., Banerjee, S., Sirmans, C.F., Tu, Y., Ong, S.E., 2007. Multilevel modelling using spatial processes: application to the Singapore housing market. Comput. Statist. Data Anal., this issue, doi:10.1016/j.csda.2006.11.019.

Geweke, J.F., 2005. Contemporary Bayesian Econometrics and Statistics. Wiley, New York.

Geweke, J., 2007. Interpretation and inference in mixture models: simple MCMC works. Comput. Statist. Data Anal., this issue, doi:10.1016/j.csda.2006.11.026.

Gilli, M., Winker, P., 2003. A global optimization heuristic for estimating agent based models. Comput. Statist. Data Anal. 42 (3), $299-312$.

Gouriéroux, C., Monfort, A., 1996. Simulation-Based Econometric Methods. Oxford University Press, Oxford.

Gülpinar, N., Rustem, B., 2007. Robust optimal decisions with imprecise forecasts. Comput. Statist. Data Anal., this issue, doi:10.1016/j.csda.2006.11.036.

Heij, C., Groenen, P., van Dijk, D., 2007. Forecast comparison of principal component regression and principal covariate regression. Comput. Statist. Data Anal., this issue, doi:10.1016/j.csda.2006.10.019.

Jung, R.C., Kukuk, M., Liesenfeld, R., 2006. Time series of count data: modelling, estimation and diagnostics. Comput. Statist. Data Anal. 51 (4), 2350-2364.

Kim, M.S., Wang, S., 2006. On the applicability of a stochastic volatility (SV) model and an SV model with jumps for some U.S. T-bill yields data. Comput. Statist. Data Anal. 51 (4), 2210-2217.

Koop, G., 2003. Bayesian Econometrics. Wiley, Chichester. 
Nankervis, J., 2005. Computational algorithms for double bootstrap confidence intervals. Comput. Statist. Data Anal. 49 (2), $461-475$.

Sanford, A.D., Martin, G.M., 2005. Simulation-based Bayesian estimation of affine term structure models. Comput. Statist. Data Anal. 49 (2), $527-554$.

Train, K.E., 2003. Discrete Choice Methods with Simulation. Cambridge University Press, Cambridge.

John Geweke

University of Iowa

E-mail address: john-geweke@uiowa.edu

Patrick J.F. Groenen

Erasmus University Rotterdam

E-mail address: groenen@few.eur.nl

Richard Paap

Erasmus University Rotterdam

E-mail address: paap@few.eur.nl

Herman K. van Dijk

Erasmus University Rotterdam

E-mail address: hkvandijk@few.eur.nl 